\title{
Decay-rate power-law exponent as a link between dissociation energy and temperature
}

\author{
Paul Fischer* and Lutz Schweikhard \\ Institut für Physik, Universität Greifswald, 17487 Greifswald, Germany
}

(Received 13 August 2020; accepted 5 October 2020; published 3 November 2020)

\begin{abstract}
Indium-cluster anions $\operatorname{In}_{n}^{-}$are probed for delayed dissociation by photoexcitation in a multi-reflection time-offlight device. In addition to prompt dissociation with below-microsecond decay constants, we observe reactions on timescales of several tens to hundreds of microseconds. These time-resolved decay-rate measurements reveal a power-law behavior in time which can be traced back to the clusters' energy distribution due to their production by laser ablation in high vacuum. Modeling energy distributions from such a production allows us to connect the cluster-specific dissociation energy with the ensemble temperature through experimentally determined powerlaw exponents.
\end{abstract}

DOI: 10.1103/PhysRevResearch.2.043177

\section{INTRODUCTION}

Delayed reactions offer fascinating approaches to the behavior and properties of nanoparticles [1]. The time-resolved study of thermionic emission of electrons [2-8] and atoms $[8,9]$ as well as that of radiative cooling $[10,11]$ has proven to be a valuable tool, yielding information on dissociation and ionization energies and the evolution of statistical processes towards the bulk [12]. One of the most intriguing discoveries is the fact that the decay rate of cluster ensembles with broad distributions of internal energies shows a power-law behavior in time, $R(t)=R_{0} t^{p}$, with initial rate $R_{0}$ and exponent $p$ [13-20]. For a sufficiently smooth and broad distribution, $p=-1$ is expected, explained by the superposition of many equally populated fractions of the cluster ensemble with different exponential decay constants. Deviations from this value can be linked to, e.g., decay-channel competition [13] or the freezing of vibrational degrees of freedom [21]. Generally, however, the power-law exponent is affected by the energy distribution and thus the ion production [22].

Investigations of delayed decays often use ion storage rings to probe molecules over extended timescales. Next to these devices and magnetic-field-based Penning traps, electrostatic ion beam traps (EIBTs) have been applied [23,24]. They are also known as (high-precision) multi-reflection time-of-flight (MR-ToF) mass spectrometers [25], particularly since their application in low-energy nuclear physics [26]. In MR-ToF mode, ion-bunch structures are preserved, and mass resolving powers quickly increase to values on the order of 100000 .

As with other ion traps [27,28], time-resolved measurements can be performed in MR-ToF devices by varying some

\footnotetext{
*Corresponding author: paul.fischer@uni-greifswald.de

Published by the American Physical Society under the terms of the Creative Commons Attribution 4.0 International license. Further distribution of this work must maintain attribution to the author(s) and the published article's title, journal citation, and DOI.
}

delay time after ion excitation and prior to ejection towards a detector [29]. For this approach the signal of each delay has to be recorded individually. In contrast, neutrals resulting from fragmentation or electron detachment traverse the mirror potentials and can be used to track the decay of excited ions revolving between the mirrors $[16,20,30,31]$. This enables the recording of decay rates over time from a single injected particle bunch. In electrostatic storage rings, the MR-ToF mass spectrometers' larger siblings, analogous studies succeeded in resolving low-lying rotational states and monitoring their long-term cooling behavior [32,33]. For these studies, however, the storage devices have been operated in "nonbunching mode," i.e., purely as electrostatic traps. Ignoring the MR-ToF trait of high mass resolving powers, precursor preparation relied on external mass-spectrometry steps.

Here we report time-resolved decay-rate measurements in bunched MR-ToF operation. Precursors are selected in an MR-ToF mass spectrometer and subsequently photoexcited without immediate ejection. For delayed dissociation channels, in-flight fragmentation leads to a shift of the fragments' kinetic energy and thus their revolution period. This allows monitoring of the charged fragments for several precursor laps in a single ToF spectrum. The resulting time-resolved fragmentation rates deviate from the $p=-1$ law, which is used to connect the cluster-ensemble temperature and the species of interest's dissociation threshold via a simple statistical model.

\section{EXPERIMENTAL SETUP}

The setup (Fig. 1) incorporates a high-vacuum laserablation source and an MR-ToF analyzer. The components are connected perpendicularly via a quadrupole deflector to accommodate two axes for the source- and excitation-laser beams (Nd:YAG with $\lambda=532 \mathrm{~nm}$ ). Note that ions are not cooled by aggregation gas as used for the production of larger clusters [34], i.e., their internal energy is largely governed by their production in the hot laser spot. They are accelerated to $2010 \mathrm{eV}$ and stored between the electrostatic mirrors by lowering their energy to $1210 \mathrm{eV}$ with an in-trap lift [35]. 


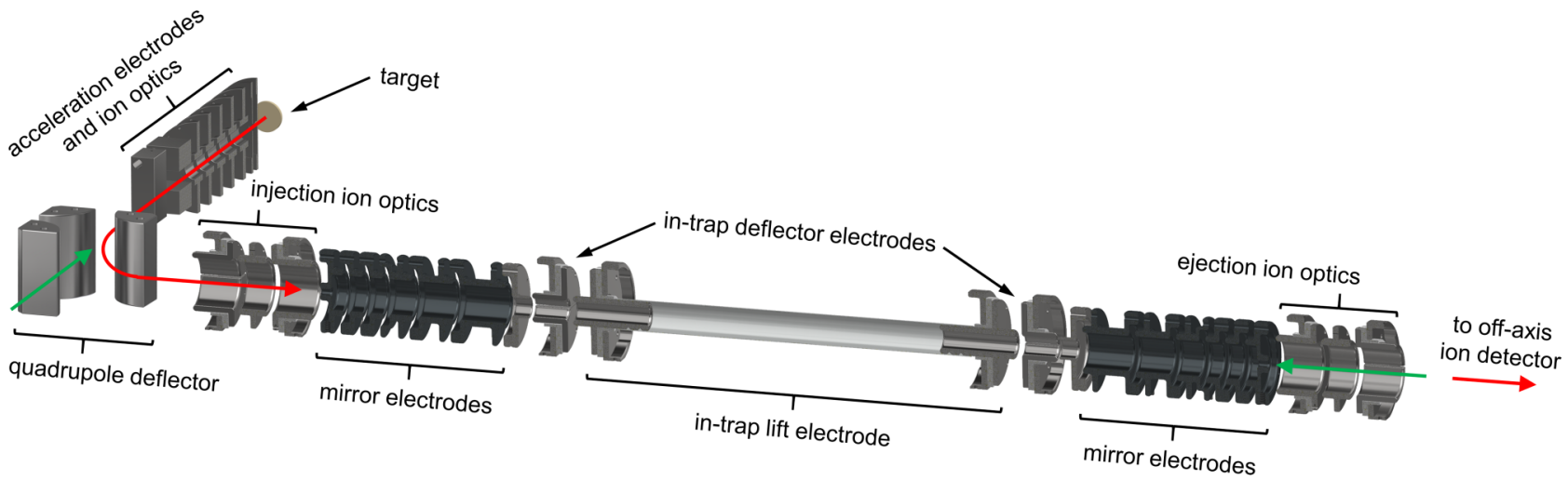

FIG. 1. Experimental setup including laser-ablation source and MR-ToF analyzer. The ion flight path is indicated in red, the source- and excitation-laser beams in green.

Unwanted ions are discarded from the trap by synchronized switching of in-trap deflector voltages [36].

In-trap photodissociation and its analysis has been described recently [37,38]. In short, a laser pulse is timed to excite the mass-selected precursors at their turnaround point in the entry-side ion mirror. Two decay timescales can be distinguished: For "prompt" dissociations $(\tau \lesssim 1 \mu \mathrm{s})$ at the turnaround point at kinetic energies of (close to) zero, the fragments retain the precursor energy. Delayed dissociation ( $\tau \gtrsim 10 \mu \mathrm{s}$ ), however, happens during the precursor revolutions between the mirrors. Fragments produced during the precursor drifts through the field-free in-trap lift electrode stay together as a bunch and retain a kinetic energy $E_{\text {kin,f }}=$ $\left(m_{\mathrm{f}} / m_{\mathrm{p}}\right) E_{\mathrm{kin}, \mathrm{p}}$ according to the ratio of their mass $m_{\mathrm{f}}$ to that of the precursors, $m_{\mathrm{p}}$. Therefore their revolution period is shifted against that of the prompt fragments.

Before discussing the delayed dissociations, an overview of the prompt reactions is given for the present case of small anionic indium clusters. This serves to highlight the dissociation channels at hand and identify species of interest for time-resolved studies.

\section{PHOTODISSOCIATION OF SMALL INDIUM CLUSTERS}

Figure 2(b) shows an example of prompt product ions of $\mathrm{In}_{12}^{-}$. To increase resolution, ions complete one additional lap after the excitation/dissociation before the exit-side mirror is opened for detection. Since all species have the same energy, their flight times scale with the mass-to-charge ratio and mass calibration is simple. The relative fragment abundances as well as branching ratios between electron detachment and dissociation of precursors $\mathrm{In}_{n}^{-}, n=2-20$, are shown in Fig. 3 . While neutral and cationic indium clusters have been the focus of previous investigations [39-43], anions have been less so. To our knowledge, no dissociation studies have yet been performed on indium anions. However, clusters of trivalent metals are known to exhibit similar properties [44], such as dominant neutral-monomer evaporation, e.g., for $\mathrm{Al}_{n}^{-}$[45]. This is also found for $\mathrm{In}_{n}^{-}$here. A notable exception to the smooth trends follows from the increased stability of $\mathrm{In}_{13}^{-}$ due to its icosahedral geometry [46,47] and closed (jellium) electronic shell of $13 \cdot 3+1=40$ valence electrons [46-48].
Consequently, the $\mathrm{In}_{15}^{-}$precursor shows increased $\mathrm{In}_{13}^{-}$fragment abundance [Fig. 3(a), spike in purple curve]. Analogous, albeit less pronounced behaviors are observed for precursors $n=16$ (green) and 17 (blue).

Furthermore, the relative electron-detachment probability drops to zero for $\operatorname{In}_{13}^{-}$. $\operatorname{In}_{16}^{-}$shows the same behavior, although this species is not attributed with increased electron affinity. Possibly, the reduced relative electron detachment results from more favorable dissociation channels. Note that $n=10$ and 19 also show decreased detachment, which may hint at an underlying $3 n$ periodicity. We further note that the switch from electron detachment for the smallest clusters to mainly dissociation for larger ones is in line with the electron affinity of metal clusters increasing as a function of size [49].

\section{DELAYED DISSOCIATION CHANNELS}

As introduced above, dissociation processes occurring on timescales of the precursor's revolution period lead to additional fragment signals beyond those from prompt decays. This is illustrated with the $\mathrm{In}_{15}^{-}$spectrum of Fig. 2(c), which

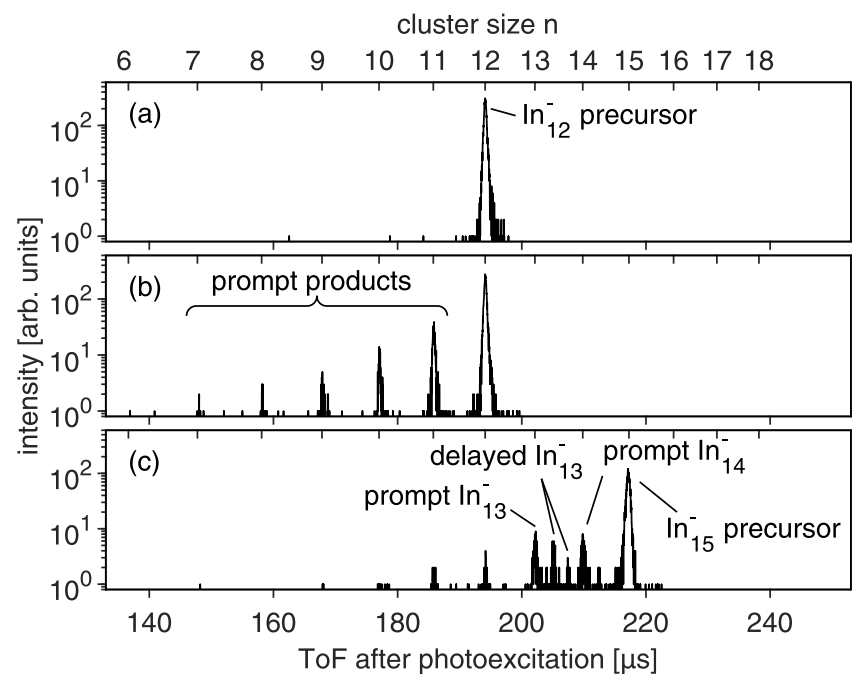

FIG. 2. Reference (a) and photodissociation spectrum of $\operatorname{In}_{12}^{-}$ (b) and $\mathrm{In}_{15}^{-}$(c) after 400 revolutions. For details, see text. 

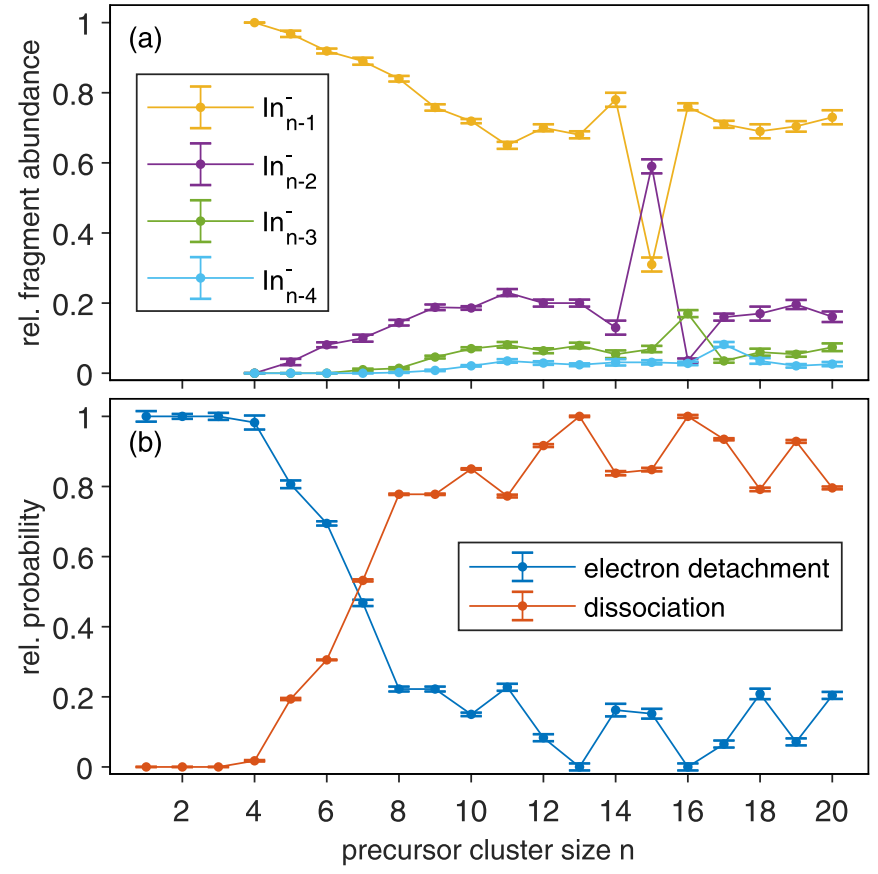

FIG. 3. (a) Relative abundances of fragment sizes $(n-1)$ through $(n-4)$ after photoexcitation of $\mathrm{In}_{n}^{-}$clusters at $\lambda=$ $532 \mathrm{~nm}$. (b) Ratio of electron detachment vs dissociation after photoexcitation.

shows two signals between $\operatorname{In}_{13}^{-}$and $\operatorname{In}_{14}^{-}$. Further investigation reveals their revolution period to be that of $\mathrm{In}_{13}^{-}$at a storage energy of $1124 \mathrm{eV}$. This shift of $86 \mathrm{eV}$ equals $13 / 14$ of the precursor storage energy, $1210 \mathrm{eV}$, which is explained by a sequential decay: The photoexcited $\mathrm{In}_{15}^{-}$precursor decays promptly by neutral-atom evaporation at the turnaround point and still has enough energy to evaporate another neutral atom. However, due to the lower remaining internal energy, this second decay is delayed and happens during the following revolution periods. The two delayed signals result from subsequent lift passes. The secondary decay products pick up additional ToF differences to the $\mathrm{In}_{14}^{-}$ions with each subsequent reflection. Thus, the earlier signal with larger difference stems from the first lift transition. Since Fig. 2 shows data from one additional post-excitation lap with photoexcitation in the entry-side mirror, there are three delayed ion bunches; however, the third is not separated from that of $\operatorname{In}_{14}^{-}$.

The shifts of the revolution period give a unique access to monitor the decay rate. Each delayed signal represents the dissociation rate at the time of the corresponding lift transition. Thus, by recording the events of several transitions, the decay rate can be monitored as a function of time in a single measurement cycle (repeated only for higher statistics). This differs from traditional studies where delay times have to be varied from one experimental cycle to another (e.g., [29]). In addition, the present scheme preserves the massspectrometric MR-ToF properties. $\mathrm{In}_{13}^{-}$is a prime candidate for these investigations due to electron detachment not being a significant competing decay channel. Also, its high fragmentation threshold yields decay lifetimes compatible to the experimental window for the present excitation photon energy, $E_{\mathrm{ph}}=2.33 \mathrm{eV}$.
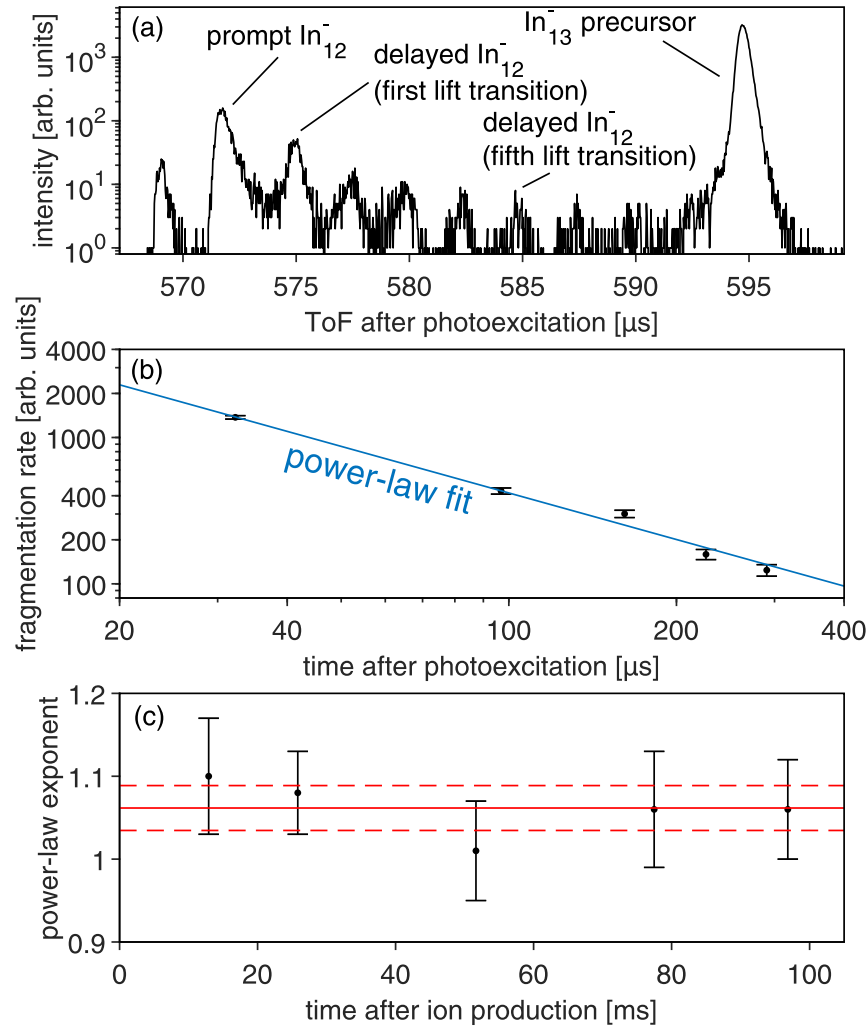

FIG. 4. (a) ToF spectrum of delayed $\mathrm{In}_{12}^{-}$signals from photoexcitation of $\mathrm{In}_{13}^{-}$after 96.84-ms flight time. The precursor is stored for four post-excitation laps (two lift transitions per lap) before ejection. (b) Ion counts of delayed $\mathrm{In}_{12}^{-}$signals and power-law fit to the data. (c) Power-law exponent from measurements after different ion flight times prior to photoexcitation. Red lines indicate weighted mean and its uncertainty.

Figure 4(a) shows a dissociation spectrum of $\operatorname{In}_{13}^{-} \rightarrow \operatorname{In}_{12}^{-}$ at four post-excitation revolutions (each revolution incurs two lift transitions). The fragmentation rate follows a power law over time [Fig. 4(b)], suggesting the presence of a broad distribution of internal energies. In the present case, such a distribution most likely results from the ions' production by laser ablation. To rule out additional cooling channels acting on the ensemble prior to the photoexcitation, the precursor flight time is varied [Fig. 4(c)]. The power-law exponent is found to be constant at $p=-1.06(3)$. It is thus assumed that the cluster ensemble is produced with a broad but (on these timescales) constant energy distribution that leads to a deviation from $p=-1$.

\section{QUANTITATIVE EVALUATION OF DELAYED DISSOCIATION}

For a quantitative consideration, the distribution is modeled with a statistical approach. To this end, the precursor ensemble is described by a single temperature $T$, i.e., all clusters are assumed to be connected to a heath bath of that temperature during their production by laser ablation. Consequently, the distribution of total vibrational energy $E$ of the ensemble follows a probability density function (PDF) $p(E)$ dependent on $T$ and their size $n$. 

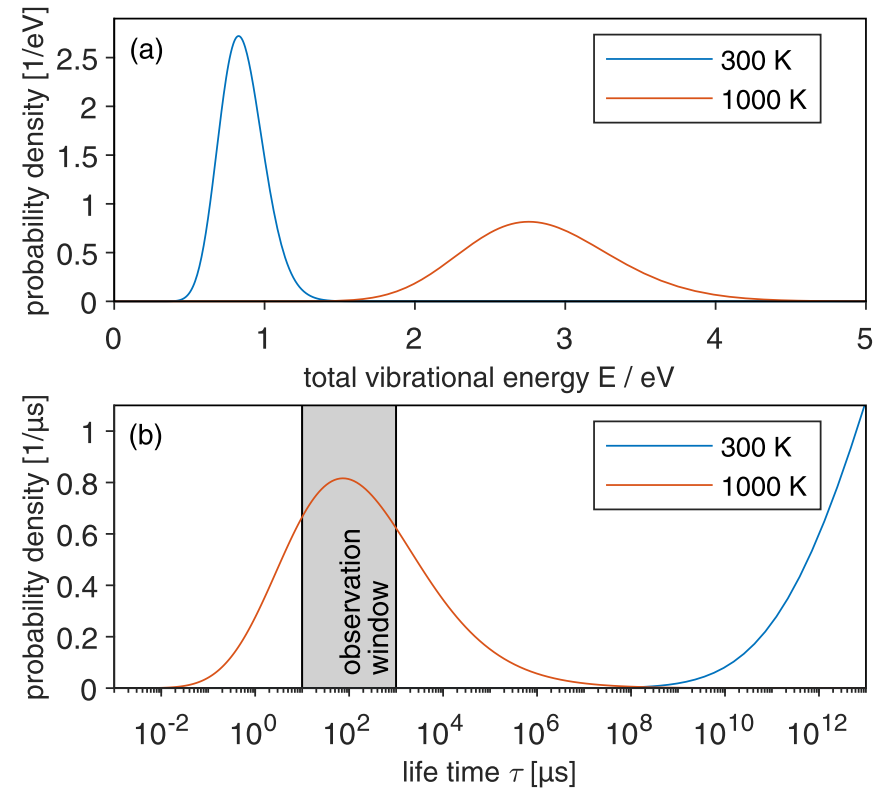

FIG. 5. (a) Theoretical distribution of total vibrational energy of an ensemble of $\operatorname{In}_{13}$ clusters ( $f=3 n-6=33$ degrees of freedom). The two examples show ensembles with temperatures $T=300 \mathrm{~K}$ (blue) and $1000 \mathrm{~K}$ (orange). (b) Cluster decay time for $D=2.64 \mathrm{eV}$ [47] and $v_{D}=129 \mathrm{~K} k_{B} / \hbar$ [50] calculated from the above energy distributions for single-photon absorption. The experimental window for observation of delayed fragmentation is shown in gray.

The PDF of such a system of oscillators is a $\Gamma$ distribution (see Appendix A),

$$
p(E)=\frac{1}{\Gamma(f)\left(k_{B} T\right)^{f}} E^{f-1} e^{-\frac{E}{k_{B} T}},
$$

with the gamma function $\Gamma(f)$, shape parameter $f$, and scaling parameter $k_{B} T$ with the Boltzmann constant $k_{B}$. This energy distribution increases with rising temperature, as exemplified in Fig. 5(a) for $T=300 \mathrm{~K}$ and $T=1000 \mathrm{~K}$. Its mean value is $f k_{B} T$; its width increases roughly with $\sqrt{f}$ (see Appendix A) and quickly spans several electronvolts for elevated temperatures.

Once the clusters are removed from the heat bath and accelerated into high vacuum, they are particles with their individual total vibrational energies still governed by $p(E)$. The clusters are modeled as microcanonical systems of $f$ classical harmonic oscillators, where $f=3 n-6$ is the number of their vibrational modes.

For the cluster energies after laser ablation $E_{\text {int }}=E+$ $n_{\mathrm{ph}} E_{\mathrm{ph}}$, the energy of $n_{\mathrm{ph}}$ absorbed photons has to be added. This energy and the dissociation energy $D$ determine the decay constant

$$
\tau=\frac{1}{v_{D} S}\left(\frac{E_{\mathrm{int}}}{E_{\mathrm{int}}-D}\right)^{f-1},
$$

as approximated by the RRK (Rice-Ramsperger-Kassel) approach $[51,52]$. Here, the Debye frequency $v_{D}$ approximates the rate of energy redistribution within the vibrational modes and $s$ is the reaction degeneracy, i.e., for monomer evaporation, the number of surface atoms. More refined theories are beyond the scope of the present study. However, the cur-

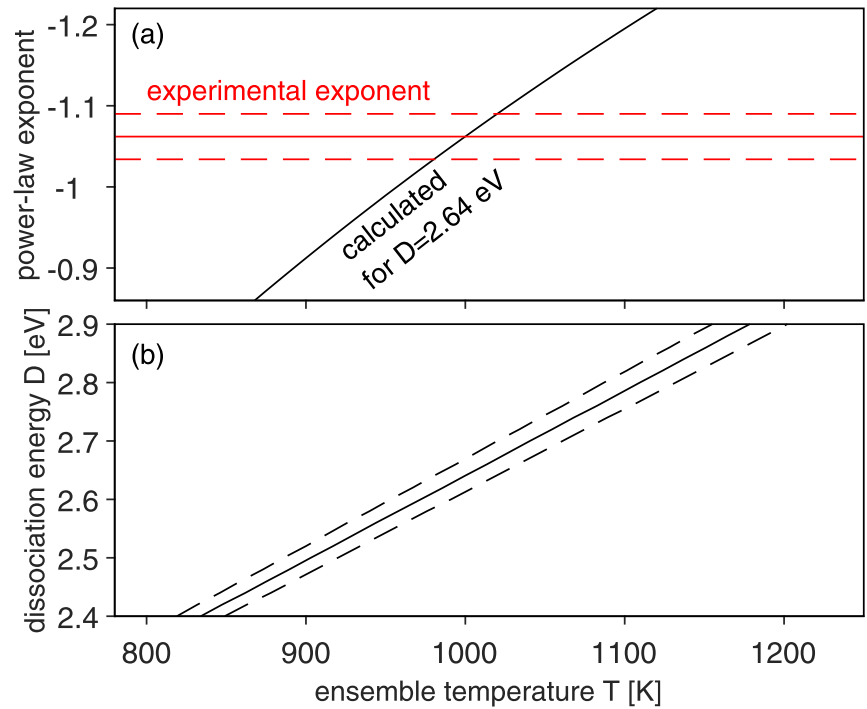

FIG. 6. (a) Calculated power-law exponent for $\mathrm{In}_{13}^{-}$cluster ensembles as a function of temperature for $D=2.64 \mathrm{eV}$ (black line); experimental $p$ value in red. (b) Dissociation energies as a function of ensemble temperature for experimental power-law exponent $p=-1.06(3)$.

rent approach is sufficient to demonstrate the principle of the method.

The dissociation lifetimes calculated based on the PDFs (1) span several orders of magnitude [Fig. 5(b)]. For comparison to the experimental data, however, the observation window has to be considered. As stated above, short decay times will only contribute to the prompt fragment signal. On the other hand, different fragment species will begin to lap one another after a few post-excitation revolutions, significantly complicating the determination of time-resolved decay rates. For the present setup and mass range, the observation window for delayed dissociation is roughly $10 \mu \mathrm{s} \lesssim \tau \lesssim 1 \mathrm{~ms}$.

The number of expected decay events during a lift transition of the cluster bunch as a function of time after the laser excitation can be calculated based on the curve shown in Fig. 5(b) and fitted by a power-law function in the experimental window (see Appendix B). Depending on which part of the distribution is probed, the power-law exponent deviates from -1 and varies monotonously with the ensemble temperature [Fig. 6(a)]. It is thus possible to relate this temperature to $p$. More precisely, since the calculated results are sensitive to the dissociation energy, $T$ and $D$ can be adjusted with respect to one another [Fig. 6(b)]. To our knowledge, the dissociation threshold of $\mathrm{In}_{13}^{-}$has not yet been determined experimentally. For a value $D=2.64 \mathrm{eV}$ based on DFT calculations [47], a temperature $T=1000(20) \mathrm{K}$ is found. However, if ensemble temperatures are obtained by other means, i.e., via species with precisely known $D$ serving as references, the method at hand can be used to directly determine cluster dissociation energies.

Note that a deviation from $p=-1$ could in theory also result from competing decay pathways [13]. However, electron emission has been found as virtually nonexistent for the present case [see Fig. 3(b)], and radiative cooling should not be a significant factor on these timescales. 
Thus, the power-law exponent measured by exploiting revolution-period shifts in the MR-ToF device connects a cluster ensemble's temperature and dissociation threshold. This allows experimental determination of either if the other one is known. The method is applicable for molecules, clusters, and other nanoparticles. An obvious extension of the present measurements is the additional scanning of the excitation photon energy to access further indium-cluster sizes as well as other cluster species.

\section{ACKNOWLEDGMENTS}

The authors thank A. Alvermann for helpful discussions concerning the description of harmonic-oscillator ensembles. The authors acknowledge support from the DFG (German Research Foundation, No. 393148499) and the Open Access Publication Fund of the University of Greifswald.

\section{APPENDIX A: THE PROBABILITY DENSITY FUNCTION OF AN ENSEMBLE OF HOT CLUSTERS}

The probability density function (PDF) of finding a cluster of size $n$ at a total internal energy $E$ in a thermalized ensemble at temperature $T$ in the classical limit is reviewed. It is assumed that the internal energy of the cluster is statistically distributed in its $f=3 n-6$ vibrational modes. Starting with the probability of a single quantum harmonic oscillator, the classical limit is derived. The total energy in $f$ modes follows from recursively adding more oscillators.

\section{Probability of one quantum harmonic oscillator}

The probability $p(n)$ of finding a quantum harmonic oscillator (with angular frequency $\omega$ ) coupled to a heat bath of temperature $T$ in the state with quantum number $n$ and energy

$$
E_{n}=\hbar \omega\left(n+\frac{1}{2}\right)
$$

is given by the Boltzmann factor [53]

$$
p(n)=\frac{1}{Z_{1}} e^{-\left(n+\frac{1}{2}\right) \frac{\hbar \omega}{k_{B} T}},
$$

where $k_{B}$ is the Boltzmann constant and the partition function

$$
Z_{1}=\sum_{n=0,1, \ldots} e^{-\frac{E_{n}}{k_{B} T}}
$$

acts as a normalization constant. Making use of the geometric series, (A3) becomes

$$
\begin{aligned}
Z_{1} & =e^{-\frac{1}{2} \frac{\hbar \omega}{k_{B} T}} \sum_{n=0,1, \ldots}\left(e^{-\frac{\hbar \omega}{k_{B} T}}\right)^{n} \\
& =e^{-\frac{1}{2} \frac{\hbar \omega}{k_{B} T}} \frac{1}{1-e^{-\frac{\hbar \omega}{k_{B} T}}}
\end{aligned}
$$

and the PDF can be rewritten as

$$
p(n)=e^{-n \frac{\hbar \omega}{k_{B} T}}\left(1-e^{-\frac{\hbar \omega}{k_{B} T}}\right) .
$$

\section{High-temperature limit}

For $k_{B} T \gg \hbar \omega$ (classical limit), the Boltzmann factor can be Taylor expanded,

$$
e^{-\frac{\hbar \omega}{k_{B} T}}=1-\frac{\hbar \omega}{k_{B} T}+\cdots
$$

and the sum

$$
\sum_{n=n_{a}}^{n_{b}} p(n)=\sum_{n=n_{a}}^{n_{b}} \frac{\hbar \omega}{k_{B} T} e^{-\frac{n \hbar \omega}{k_{B} T}}
$$

for the probability to find the oscillator in any state $n$ with $n_{a} \leqslant n \leqslant n_{b}$, i.e., at an energy $E$ with $E_{a} \leqslant E \leqslant E_{b}$, becomes

$$
\int_{E_{a}}^{E_{b}} p(E) d E=\frac{1}{k_{B} T} \int_{E_{a}}^{E_{b}} e^{-\frac{E}{k_{B} T}} d E,
$$

with the continuous energy $E$. Thus the PDF with respect to energy of a single classical harmonic oscillator is

$$
p(E)=\frac{1}{k_{B} T} e^{-\frac{E}{k_{B} T}} .
$$

\section{Two harmonic oscillators}

Next a second oscillator with PDF $p_{0}\left(E_{0}\right)$ is added, i.e., the PDFs

$$
\begin{aligned}
& p_{1}\left(E_{1}\right)=\frac{1}{k_{B} T} e^{-\frac{E_{1}}{k_{B} T}}, \\
& p_{0}\left(E_{0}\right)=\frac{1}{k_{B} T} e^{-\frac{E_{0}}{k_{B} T}},
\end{aligned}
$$

of two oscillators are combined, with the total energy $E_{2}=$ $E_{1}+E_{0}$. Obviously, the PDF of the energy of the ensemble is

$$
\begin{aligned}
p_{2}\left(E_{2}\right) & =\int_{0}^{E_{2}} p_{1}\left(E_{1}\right) p_{0}\left(E_{0}\right) d E_{1} \\
& =\frac{1}{\left(k_{B} T\right)^{2}} \int_{0}^{E_{2}} e^{-\frac{E_{1}}{k_{B} T}} e^{-\frac{E_{0}}{k_{B} T}} d E_{1},
\end{aligned}
$$

since the PDF of the added oscillator has to be multiplied with $p_{1}\left(E_{1}\right)$ of the first. Due to $E_{1}=E_{2}-E_{0}$, this results in

$$
\begin{aligned}
p_{2}\left(E_{2}\right) & =\frac{1}{\left(k_{B} T\right)^{2}} \int_{0}^{E_{2}} e^{-\frac{E_{2}-E_{0}}{k_{B} T}} e^{-\frac{E_{0}}{k_{B} T}} d E_{1} \\
& =\frac{1}{\left(k_{B} T\right)^{2}} \int_{0}^{E_{2}} e^{-\frac{E_{2}}{k_{B} T}} d E_{1}=\frac{1}{\left(k_{B} T\right)^{2}} E_{2} e^{-\frac{E_{2}}{k_{B} T}} .
\end{aligned}
$$

Note that the integrand ends up being independent of $E_{1}$, trivializing the integration.

\section{Many harmonic oscillators}

In analogy to the above considerations, the PDF for $f$ oscillators follows from that of $f-1$ via the recurrence relation

$$
p_{f}\left(E_{f}\right)=\int_{0}^{E_{f}} p_{f-1}\left(E_{f-1}\right) p_{0}\left(E_{0}\right) d E_{f-1} .
$$


Thus, the PDF becomes

$$
\begin{aligned}
p_{f}\left(E_{f}\right) & =\frac{1}{\left(k_{B} T\right)^{f}} \int_{0}^{E_{f}} \ldots\left[\int_{0}^{E_{3}}\left[\int_{0}^{E_{2}} e^{-\frac{E_{1}}{k_{B} T}} e^{-\frac{E_{0}}{k_{B} T}} d E_{1}\right] e^{-\frac{E_{0}}{k_{B} T}} d E_{2}\right] \ldots e^{-\frac{E_{0}}{k_{B} T}} d E_{f-1} \\
& =\frac{1}{\left(k_{B} T\right)^{f}} \int_{0}^{E_{f}} \ldots\left[\int_{0}^{E_{3}}\left[\int_{0}^{E_{2}} e^{-\frac{E_{2}}{k_{B} T}} d E_{1}\right] e^{-\frac{E_{0}}{k_{B} T}} d E_{2}\right] \ldots e^{-\frac{E_{0}}{k_{B} T}} d E_{f-1} \\
& =\frac{1}{\left(k_{B} T\right)^{f}} \int_{0}^{E_{f}} \ldots\left[\int_{0}^{E_{3}} E_{2} e^{-\frac{E_{2}}{k_{B} T}} e^{-\frac{E_{0}}{k_{B} T}} d E_{2}\right] \ldots e^{-\frac{E_{0}}{k_{B} T}} d E_{f-1} \\
& =\frac{1}{\left(k_{B} T\right)^{f}} \int_{0}^{E_{f}} \ldots\left[\int_{0}^{E_{3}} E_{2} e^{-\frac{E_{3}}{k_{B} T}} d E_{2}\right] \ldots e^{-\frac{E_{0}}{k_{B} T}} d E_{f-1} \\
& =\frac{1}{\left(k_{B} T\right)^{f}} \int_{0}^{E_{f}} \ldots\left[\frac{E_{3}^{2}}{2} e^{-\frac{E_{3}}{k_{B} T}} \ldots e^{-\frac{E_{0}}{k_{B} T}} d E_{f-1}=\cdots=\frac{1}{\left(k_{B} T\right)^{f}} e^{-\frac{E_{f}}{k_{B} T}} \frac{E_{f}^{f-1}}{(f-1) !} .\right.
\end{aligned}
$$

Again, the integrand becomes independent of all energies $E_{1}$ through $E_{f-1}$. In conclusion, the PDF with respect to the energy of an ensemble of $f$ harmonic oscillators is given by

$$
p(E)=\frac{1}{\Gamma(f)\left(k_{B} T\right)^{f}} E^{f-1} e^{-\frac{E}{k_{B} T}},
$$

where $\Gamma(f)$ is the $\Gamma$ function. Note that this PDF is a $\Gamma$ distribution, with $f$ as the shape parameter and $k_{B} T$ as the scaling parameter. The above derivation does not depend on the individual frequencies of the oscillators as long as the classical-limit assumption is applicable for all of them.

The PDFs according to (A16) are shown in Fig. 7 for several cluster sizes. While the energy distributions are

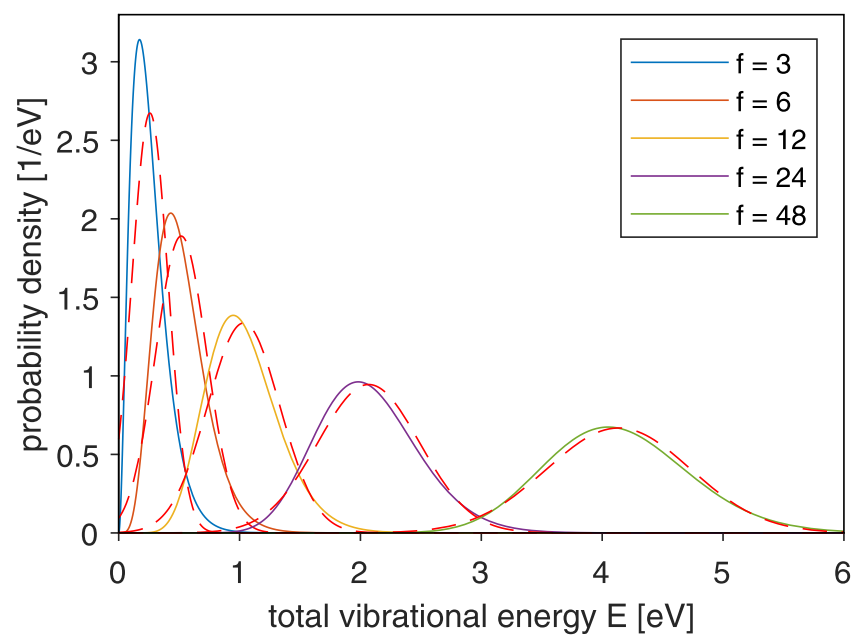

FIG. 7. Probability density of vibrational energy for cluster sizes $n=3,4,6,10,18(f=3 n-6)$ resulting from (A16) for $T=$ $1000 \mathrm{~K}$. Gaussian functions following (A17) are added for each distribution (dashed red lines). asymmetric for small $f$, they quickly become symmetrical with an increasing number of oscillators and are well approximated by Gaussian functions,

$$
p_{G}(E)=\frac{1}{k_{B} T \sqrt{2 \pi f}} \exp \left(-\frac{\left(E-f k_{B} T\right)^{2}}{2 f\left(k_{B} T\right)^{2}}\right),
$$

with mean value $f k_{B} T$ and standard deviation $\sqrt{f} k_{B} T$ (dashed red curves). The width of the PDFs governing the cluster ensemble's vibrational energy thus increases with $\sqrt{f}$.

\section{APPENDIX B: POWER-LAW EXPONENT AS A FUNCTION OF ENSEMBLE TEMPERATURE}

Following Refs. [13,15], the unimolecular decay with a power-law behavior is linked to a broad, smooth distribution of a molecular ensemble's internal energies. Thus, a number of clusters with different lifetimes and occupation densities are present, adding up to a total decay rate with $\sim t^{p}$ characteristic. For equally spaced and equally populated cluster energies, $p=-1$ is expected [15].

Three curves resulting from (A16) for cluster size $n=13$ $(f=33)$ are shown in Fig. 8(a) for different ensemble temperatures. According to the RRK (Rice-Ramsperger-Kassel) approach [51,52], the dissociation lifetimes are

$$
\tau=\frac{1}{v_{D} S}\left(\frac{E_{\mathrm{int}}}{E_{\mathrm{int}}-D}\right)^{f-1},
$$

where $E_{\mathrm{int}}=E+n_{\mathrm{ph}} E_{\mathrm{ph}}$ is a molecule's total internal energy after photoexcitation, $D$ is the dissociation energy, $s$ is the decay degeneracy, and $v_{D}$ is the rate of energy redistribution approximated by the Debye frequency. The $\tau$ distributions of $\mathrm{In}_{13}^{-}$resulting from three ensemble temperatures are shown in Fig. 8(b). 

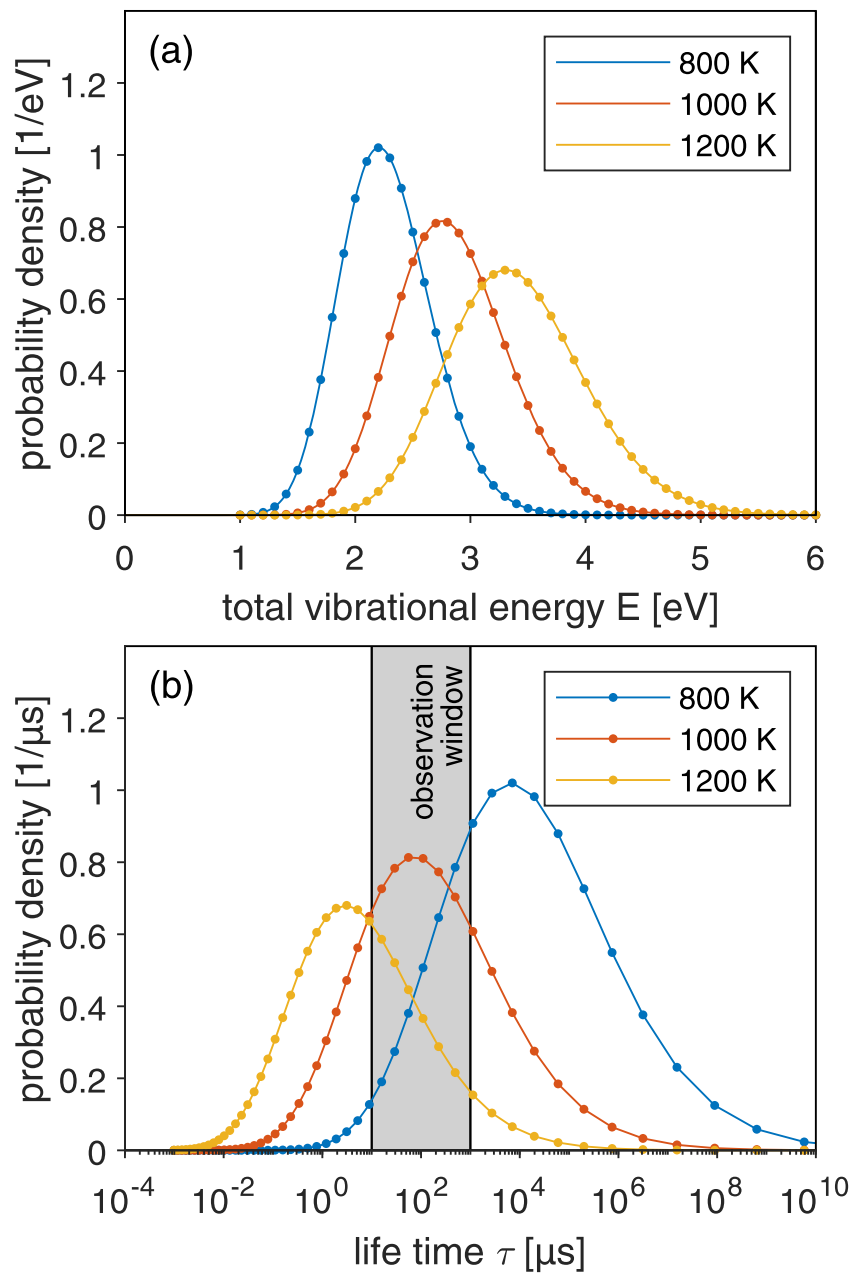

FIG. 8. (a) Distributions of vibrational energy for cluster size $n=13$ for $T=800,1000,1200 \mathrm{~K}$. (b) Distribution of lifetimes with respect to loss of a neutral monomer $\left(D=2.64 \mathrm{eV}\right.$ [47], $v_{D}=$ $129 \mathrm{~K} k_{B} / \hbar$ [50]) resulting from the energy distributions of (a) and additional photoexcitation with photon energy $2.33 \mathrm{eV}$.
Akin to Fig. 5(b), the experimental observation window for delayed dissociation is indicated in gray. Note that different temperatures lead to different parts of the distribution being probed by the window: for $800 \mathrm{~K}$, clusters with longer decay times exhibit higher probability densities; for $1200 \mathrm{~K}$, shorter decay times show higher values. For $1000 \mathrm{~K}$, there is only a slight change in probability density over the range of the observation window. For each distribution, a series of discrete sampling points with equidistant energy steps of $100 \mathrm{meV}$ is marked by the dots in Fig. 8. These yield pairs of decay lifetimes $\tau_{i}$ and corresponding probability densities $p_{i}$. Thus, decay rates

$$
R_{i}=\frac{p_{i}}{\tau_{i}} e^{-\frac{t}{\tau_{i}}}
$$

can be calculated for each section of the distribution (red lines in Fig. 9).

The sum of these individual decay rates (blue, orange, and yellow lines in Fig. 9) form the total rate $R$ accessible to the measurement. Although its actual behavior over time is nontrivial, a power-law function

$$
R(t)=R_{0} t^{p}
$$

with some initial rate $R_{0}$ and exponent $p$ can be fitted within the limited range of the observation window (black lines in Fig. 9; note that power laws form straight lines on log-log scales).

Obviously, the value of the exponent $p$ depends on the $\tau$ distribution probed by the observation window: In the $800-\mathrm{K}$ case, where decays with longer lifetimes are more abundant, the overall slope of the power law is flattened, resulting in a value $|p|<1$. Analogously, a steeper curve with $|p|>1$ results for $1200 \mathrm{~K}$. For (roughly) equal populations in the observation window, as in the case of the $1000-\mathrm{K}$ ensemble, a value of $p$ close to -1 is found, as expected [15].

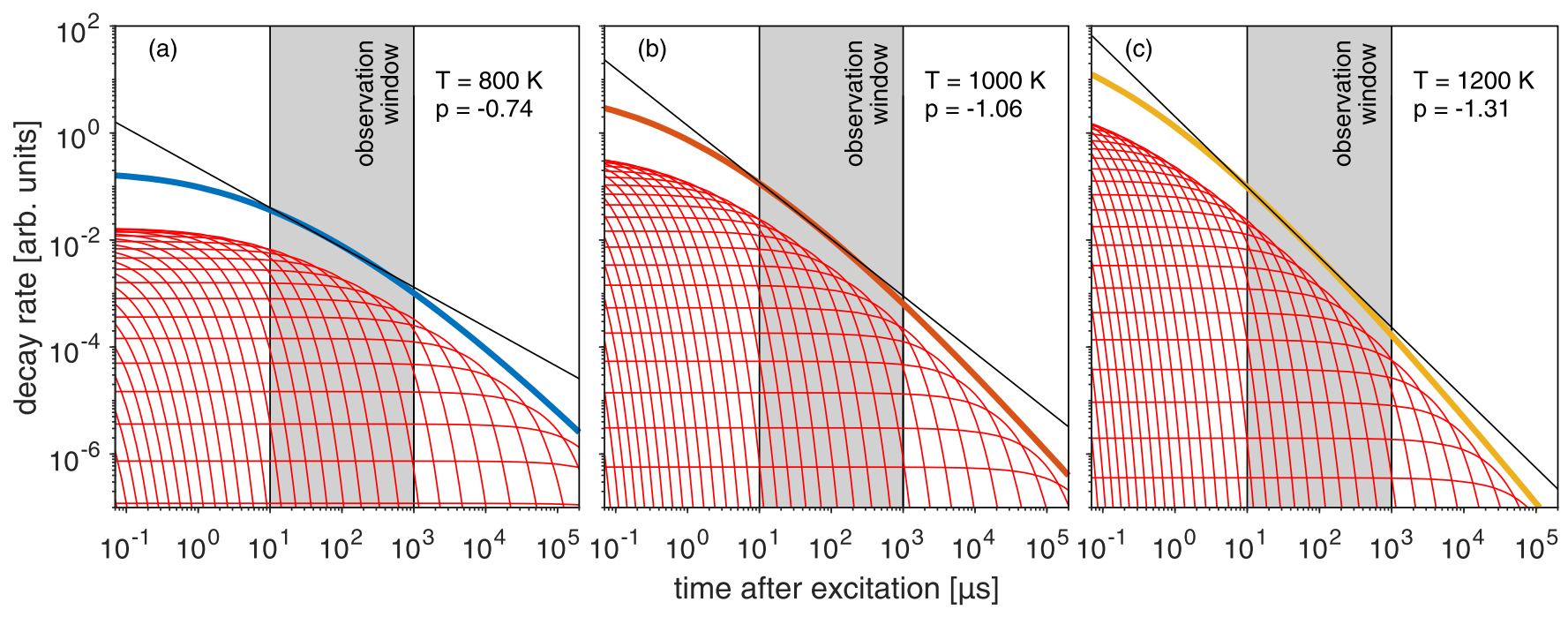

FIG. 9. Individual decay rates calculated from the lifetime distributions shown in Fig. 8 (red lines) and total sum of all rates (blue, orange, and yellow lines). A power-law curve is fitted to the sum in the experimental observation window (black lines). 
[1] E. E. B. Campbell and R. D. Levine, Delayed ionization and fragmentation en route to thermionic emission: Statistics and dynamics, Annu. Rev. Phys. Chem. 51, 65 (2000).

[2] A. Amrein, R. Simpson, and P. Hackett, Delayed ionization following photoexcitation of small clusters of refractory elements: Nanofilaments, J. Chem. Phys. 94, 4663 (1991).

[3] E. E. B. Campbell, G. Ulmer, and I. V. Hertel, Delayed Ionization of $\mathrm{C}_{60}$ and $\mathrm{C}_{70}$, Phys. Rev. Lett. 67, 1986 (1991).

[4] L.-S. Wang, J. Conceicao, C. Jin, and R. E. Smalley, Threshold photodetachment of cold $\mathrm{C}_{60}^{-}$, Chem. Phys. Lett. 182, 5 (1991).

[5] T. Leisner, K. Athanassenas, D. Kreisle, E. Recknagel, and O. Echt, Thermionic emission from free, photoexcited tungsten clusters, J. Chem. Phys. 99, 9670 (1993).

[6] D. Ding, J. Huang, R. N. Compton, C. E. Klots, and R. E. Haufler, $\mathrm{CW}$ Laser Ionization of $\mathrm{C}_{60}$ and $\mathrm{C}_{70}$, Phys. Rev. Lett. 73, 1084 (1994).

[7] H. Weidele, S. Becker, H.-J. Kluge, M. Lindinger, L. Schweikhard, C. Walther, J. Ziegler, and D. Kreisle, Delayed electron emission of negatively charged tungsten clusters, Surf. Rev. Lett. 03, 541 (1996).

[8] Y. Shi, V. A. Spasov, and K. M. Ervin, Competitive fragmentation and electron loss kinetics of photoactivated silver cluster anions: Dissociation energies of $\mathrm{Ag}_{n}^{-}(n=7-11)$, J. Chem. Phys. 111, 938 (1999).

[9] A. Amrein, R. Simpson, and P. Hackett, Multiphoton excitation, ionization, and dissociation decay dynamics of small clusters of niobium, tantalum, and tungsten: Time-resolved thermionic emission, J. Chem. Phys. 95, 1781 (1991).

[10] U. Frenzel, U. Kalmbach, D. Kreisle, and E. Recknagel, Laserinduced optical-emission studies of niobium clusters, Surf. Rev. Lett. 03, 505 (1996).

[11] C. Walther, G. Dietrich, W. Dostal, K. Hansen, S. Krückeberg, K. Lützenkirchen, and L. Schweikhard, Radiative Cooling of a Small Metal Cluster: The Case of $V_{13}^{+}$, Phys. Rev. Lett. 83, 3816 (1999).

[12] K. Hansen, Statistical Physics of Nanoparticles in the Gas Phase (Springer, Netherlands, 2013).

[13] K. Hansen and O. Echt, Thermionic Emission and Fragmentation of $\mathrm{C}_{60}$, Phys. Rev. Lett. 78, 2337 (1997).

[14] J. U. Andersen, C. Gottrup, K. Hansen, P. Hvelplund, and M. Larsson, Radiative cooling of fullerene anions in a storage ring, Eur. Phys. J. D 17, 189 (2001).

[15] K. Hansen, J. U. Andersen, P. Hvelplund, S. P. Møller, U. V. Pedersen, and V. V. Petrunin, Observation of a $1 / t$ Decay Law for Hot Clusters and Molecules in a Storage Ring, Phys. Rev. Lett. 87, 123401 (2001).

[16] M. W. Froese, K. Blaum, F. Fellenberger, M. Grieser, M. Lange, F. Laux, S. Menk, D. A. Orlov, R. Repnow, T. Sieber, Y. Toker, R. von Hahn, and A. Wolf, Thermionic power-law decay of excited aluminum-cluster anions and its dependence on storagedevice temperature, Phys. Rev. A 83, 023202 (2011).

[17] M. Lange, M. W. Froese, S. Menk, D. Bing, F. Fellenberger, M. Grieser, F. Laux, D. A. Orlov, R. Repnow, T. Sieber, Y. Toker, R. von Hahn, A. Wolf, and K. Blaum, Radiative cooling of $\mathrm{Al}_{4}^{-}$ and $\mathrm{Al}_{5}^{-}$in a cryogenic environment, New J. Phys. 14, 065007 (2012).

[18] M. Goto, A. E. K. Sundén, H. Shiromaru, J. Matsumoto, H. Tanuma, T. Azuma, and K. Hansen, Direct observation of internal energy distributions of $\mathrm{C}_{5}^{-}$, J. Chem. Phys. 139, 054306 (2013).
[19] S. Martin, J. Bernard, R. Brédy, B. Concina, C. Joblin, M. Ji, C. Ortega, and L. Chen, Fast Radiative Cooling of Anthracene Observed in a Compact Electrostatic Storage Ring, Phys. Rev. Lett. 110, 063003 (2013).

[20] C. Breitenfeldt, K. Blaum, M. W. Froese, S. George, G Guzmán-Ramírez, M. Lange, S. Menk, L. Schweikhard, and A. Wolf, Decay processes and radiative cooling of small anionic copper clusters, Phys. Rev. A 94, 033407 (2016).

[21] S. Menk, S. Das, K. Blaum, M. W. Froese, M. Lange, M. Mukherjee, R. Repnow, D. Schwalm, R. von Hahn, and A. Wolf, Vibrational autodetachment of sulfur hexafluoride anions at its long-lifetime limit, Phys. Rev. A 89, 022502 (2014).

[22] K. Hansen, Decay dynamics in molecular beams, Mass Spectrom. Rev. 21630, 1 (2020).

[23] W. H. Benner, A gated electrostatic ion trap to repetitiously measure the charge and $\mathrm{m} / \mathrm{z}$ of large electrospray ions, Anal. Chem. 69, 4162 (1997).

[24] D. Zajfman, O. Heber, L. Vejby-Christensen, I. Ben-Itzhak, M. Rappaport, R. Fishman, and M. Dahan, Electrostatic bottle for long-time storage of fast ion beams, Phys. Rev. A 55, R1577 (1997).

[25] H. Wollnik and M. Przewloka, Time-of-flight mass spectrometers with multiply reflected ion trajectories, Int. J. Mass Spectrom. 96, 267 (1990).

[26] F. Wienholtz, D. Beck, K. Blaum, C. Borgmann, M. Breitenfeldt, R. B. Cakirli, S. George, F. Herfurth, J. D. Holt, M. Kowalska, S. Kreim, D. Lunney, V. Manea, J. Menendez, D. Neidherr, M. Rosenbusch, L. Schweikhard, A. Schwenk, J. Simonis, J. Stanja et al., Masses of exotic calcium isotopes pin down nuclear forces, Nature (London) 498, 346 (2013).

[27] R. C. Dunbar, Photodissociation of trapped ions, Int. J. Mass Spectrom. 200, 571 (2000).

[28] M. Vogel, K. Hansen, A. Herlert, and L. Schweikhard, ModelFree Determination of Dissociation Energies of Polyatomic Systems, Phys. Rev. Lett. 87, 013401 (2001).

[29] B. Kafle, O. Aviv, V. Chandrasekaran, O. Heber, M. L. Rappaport, H. Rubinstein, D. Schwalm, D. Strasser, and D. Zajfman, Electron detachment and fragmentation of laserexcited rotationally hot $\mathrm{Al}_{4}{ }^{-}$, Phys. Rev. A 92, 052503 (2015).

[30] A. Naaman, K. G. Bhushan, H. B. Pedersen, N. Altstein, O. Heber, M. L. Rappaport, R. Moalem, and D. Zajfman, Metastable states of negative carbon clusters: $C_{n}^{-}, n=2-6$, J. Chem. Phys. 113, 4662 (2000).

[31] O. Aviv, Y. Toker, D. Strasser, M. L. Rappaport, O. Heber, D. Schwalm, and D. Zajfman, Competition between delayed ionization and fragmentation of laser-excited $\mathrm{Al}_{4}^{-}$, Phys. Rev. A 83, 023201 (2011).

[32] C. Meyer, A. Becker, K. Blaum, C. Breitenfeldt, S. George, J. Göck, M. Grieser, F. Grussie, E. A. Guerin, R. von Hahn, P. Herwig, C. Krantz, H. Kreckel, J. Lion, S. Lohmann, P. M. Mishra, O. Novotný, A. P. O’Connor, R. Repnow, S. Saurabh et al., Radiative Rotational Lifetimes and State-Resolved Relative Detachment Cross Sections from Photodetachment Thermometry of Molecular Anions in a Cryogenic Storage Ring, Phys. Rev. Lett. 119, 023202 (2017).

[33] H. T. Schmidt, G. Eklund, K. C. Chartkunchand, E. K. Anderson, M. Kamińska, N. de Ruette, R. D. Thomas, M. K. Kristiansson, M. Gatchell, P. Reinhed, S. Rosén, A. Simonsson, A. Källberg, P. Löfgren, S. Mannervik, H. Zettergren, and H. Cederquist, Rotationally Cold OH- Ions in the Cryogenic 
Electrostatic Ion-Beam Storage Ring DESIREE, Phys. Rev. Lett. 119, 073001(R) (2017); Erratum: Rotationally Cold $\mathrm{OH}^{-}$ Ions in the Cryogenic Electrostatic Ion-Beam Storage Ring DESIREE [Phys. Rev. Lett. 119, 073001 (2017)], 121, 079901(E) (2018).

[34] T. G. Dietz, M. A. Duncan, D. E. Powers, and R. E. Smalley, Laser production of supersonic metal cluster beams, J. Chem. Phys. 74, 6511 (1981).

[35] R. N. Wolf, G. Marx, M. Rosenbusch, and L. Schweikhard, Static-mirror ion capture and time focusing for electrostatic ionbeam traps and multi-reflection time-of-flight mass analyzers by use of an in-trap potential lift, Int. J. Mass Spectrom. 313, 8 (2012).

[36] P. Fischer, S. Knauer, G. Marx, and L. Schweikhard, In-depth study of in-trap high-resolution mass separation by transversal ion ejection from a multi-reflection time-of-flight device, Rev. Sci. Instrum. 89, 015114 (2018).

[37] P. Fischer and L. Schweikhard, Photofragmentation of $\mathrm{Bi}_{\mathrm{n}}^{+/-}$ clusters $(n=2-19)$ in an electrostatic ion beam trap, Eur. Phys. J. D 73, 105 (2019).

[38] P. Fischer and L. Schweikhard, Isotope-resolved photodissociation pathways of lead-doped bismuth clusters from tandem multi-reflection time-of-flight mass spectrometry, Phys. Rev. Res. 1, 033050 (2019).

[39] D. Rayane, P. Melinon, B. Cabaud, A. Hoareau, B. Tribollet, and M. Broyer, Electronic properties and fragmentation processes for singly and doubly charged indium clusters, J. Chem. Phys. 90, 3295 (1989).

[40] B. Baguenard, M. Pellarin, C. Bordas, J. Lermé, J. Vialle, and M. Broyer, Shell structure of small indium clusters below $\mathrm{N} \approx$ 200 atoms, Chem. Phys. Lett. 205, 13 (1993).

[41] Z. Ma, S. R. Coon, W. F. Calaway, M. J. Pellin, D. M. Gruen, and E. I. von Nagy-Felsobuki, Sputtering of neutral and ionic indium clusters, J. Vac. Sci. Technol. A 12, 2425 (1994).
[42] C. Staudt and A. Wucher, Generation of large indium clusters by sputtering, Phys. Rev. B 66, 075419 (2002).

[43] C. Staudt, A. Wucher, S. Neukermans, E. Janssens, F. Vanhoutte, E. Vandeweert, R. Silverans, and P. Lievens, Internal excitation of sputtered neutral indium clusters, Nucl. Instrum. Methods Phys. Res., Sect. B 193, 787 (2002).

[44] F. L. King and M. M. Ross, Abundance distributions and dissociations of sputtered aluminum, gallium, and indium cluster ions, Chem. Phys. Lett. 164, 131 (1989).

[45] W. A. Saunders, P. Fayet, and L. Wöste, Photodestruction of positively and negatively charged aluminum-cluster ions, Phys. Rev. A 39, 4400 (1989).

[46] W. Zhang, G. Zhao, J. Sun, L. Zhi, and Y. Gu, First-principles study of the geometrical and electronic structures of $\operatorname{In}_{\mathrm{n}}(\mathrm{n}=$ 2-16) clusters, Chem. Phys. 361, 44 (2009).

[47] S. Shi, Y. Liu, Y. Li, B. Deng, C. Zhang, and G. Jiang, A density functional study of small neutral, anionic, and cationic indium clusters $\operatorname{In}_{n}, \operatorname{In}_{n}^{-}$, and $\operatorname{In}_{\mathrm{n}}^{+}(\mathrm{n}=2-15)$, Comput. Theor. Chem. 1079, 47 (2016).

[48] M. Gausa, G. Ganteför, H. O. Lutz, and K. H. Meiwes-Broer, Electronic level structure of group III heavy element clusters studied by photoelectron spectroscopy, Int. J. Mass Spectrom. 102, 227 (1990).

[49] R. Johnston, Atomic and Molecular Clusters (Taylor \& Francis, New York, 2002).

[50] C. Y. Ho, R. W. Powell, and P. E. Liley, Thermal conductivity of the elements, J. Phys. Chem. Ref. Data 1, 279 (1972).

[51] O. K. Rice and H. C. Ramsperger, Theories of unimolecular gas reactions at low pressures, J. Am. Chem. Soc. 49, 1617 (1927).

[52] L. S. Kassel, The distribution of energy in molecules, Proc. Nat. Acad. Sci. USA 14, 23 (1928).

[53] F. Schwabl, Statistical Mechanics (Springer-Verlag, Berlin, 2006). 